\title{
Proposal of Distance Measurement Using Ceiling Light and Two Photodiodes to Conpensationg Light Variation
}

\author{
Kanako Kinoshita ${ }^{\text {a, }}$, Shiyuan Yang ${ }^{\mathrm{a}}$, Mingyao Qi ${ }^{\mathrm{b}}$ \\ ${ }^{a}$ Department of Electrical and Electronics Engineering, Kyushu Institute of Technology, Japan \\ ${ }^{b}$ Logistics and Management Division, The Graduate School at Shenzhen, Tsinghua University \\ *Corresponding Author: kinoshita.kanako408@ mail.kyutech.jp
}

\begin{abstract}
In recent years, many researches have been performed on indoor autonomous mobile robots, and accurate self-location estimation is required for this purpose. It is necessary to have a system that makes landmarks that exist in all places and that can detect positions easily and clearly. Therefore, in this paper we propose a position estimation method using photodiodes and LED illumination.

In one of our previous method, two photodiodes and one LED lighting were used, and it depended on the maximum value of the illuminance just under the LED lighting. However, as the characteristic of LED lighting that the illuminance intensity changes depending on time, it is necessary to obtain the maximum value of illuminance at every time, but it is impossible for real applications.

In this study, we used two photodiodes to realize a method to estimate the position irrespective of the illuminance intensity variation of LED lighting. Moreover, since the system itself uses inexpensive photodiodes, it has a merit of low cost.
\end{abstract}

Keywords: LED, photodiode, indoor position detection

\section{Introduction}

In recent years, unmanned transfer robots have been used for transporting equipment and equipment in hospitals, as well as manufacturing lines for automobiles and foods. Although it is often used mainly for work such as factories and hospitals, it is thought that the place where robots will be active will expand in the future. In order to make it a robot that can help people's everyday life, it is necessary not to run only on a fixed course but to be free to travel anywhere. And in order to be able to travel freely, we have to recognize our own position accurately.

In this paper, we propose a self-location estimation method using one indoor LED illumination and two photodiodes as a system for recognizing self-location based on what already exists in every place. In addition, we propose a self-location estimation method that does not depend on the maximum value of the illuminance directly under the LED lighting, which has been a problem until now.

\section{Principle of this research system}

\subsection{Principle of illumination}

From the inverse square law of the distance with respect to the illuminance and the oblique incident light characteristic, the illuminance distribution of the plane to be illuminated by the microscopic light source such as the cosine fourth power law is used.

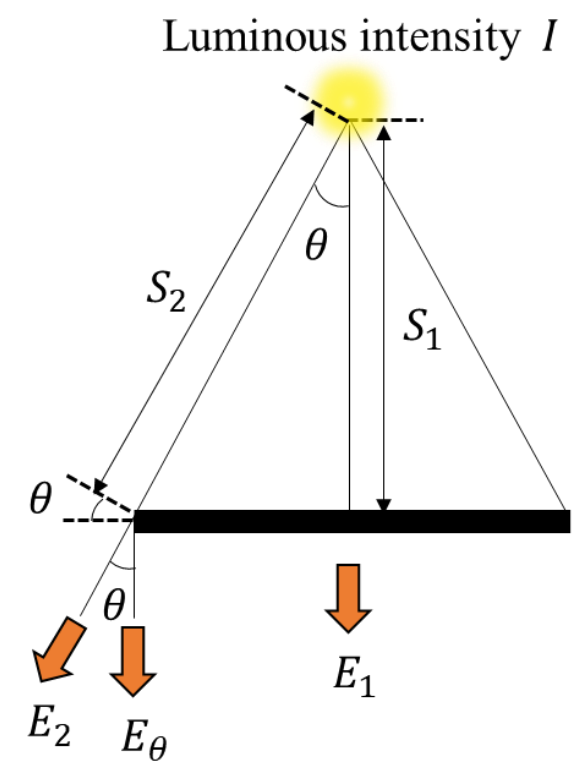

Fig. 2.1 Illuminance distribution 


$$
\begin{aligned}
& E_{1}=\frac{I}{4 \pi S_{1}^{2}} \\
& E_{2}=\frac{I}{4 \pi S_{2}^{2}}
\end{aligned}
$$

Also, from the cos fourth power law

$$
E_{\theta}=E_{1} \cos ^{4} \theta
$$

From Equation (2.3), it is possible to predict the distance from the center by using illuminance. Let the maximum illuminance immediately under the LED illumination be $E_{\max }$ which is measured previously and place the photodiode at $\mathrm{P} 1$ position in Fig. 2.2.

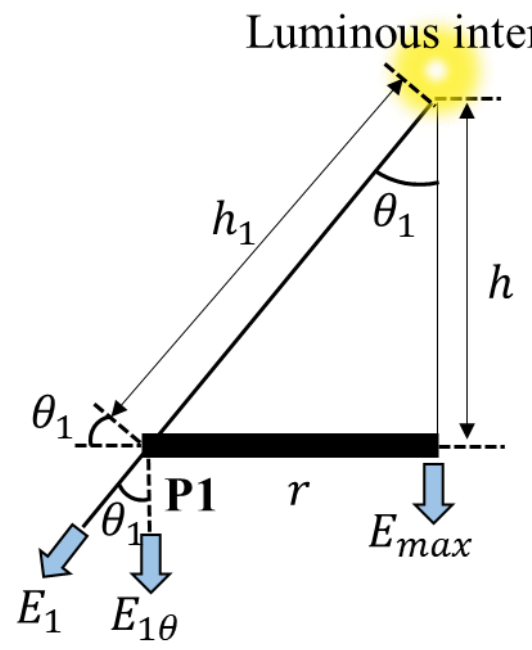

Fig. 2.2 Principle of calculating radius

From equation (2.3)

$$
\begin{gathered}
E_{1 \theta}=E_{\text {max }} \cos ^{4} \theta_{1} \\
\therefore \theta_{1}=\cos ^{-1}\left(\sqrt[4]{\frac{E_{1 \theta}}{E_{\text {max }}}}\right)
\end{gathered}
$$

Than the Pythagorean theorem

$$
\begin{gathered}
r=h \tan \theta_{1} \\
\therefore r=h \tan \left\{\cos ^{-1}\left(\sqrt[4]{\frac{E_{1 \theta}}{E_{\max }}}\right)\right\}
\end{gathered}
$$

\subsection{Principle of position detection by illuminance}

The principle of estimating the position by the illuminance shown above will be describedPlace the photodiode on P1 and P2 as shown in Fig. 2.2.

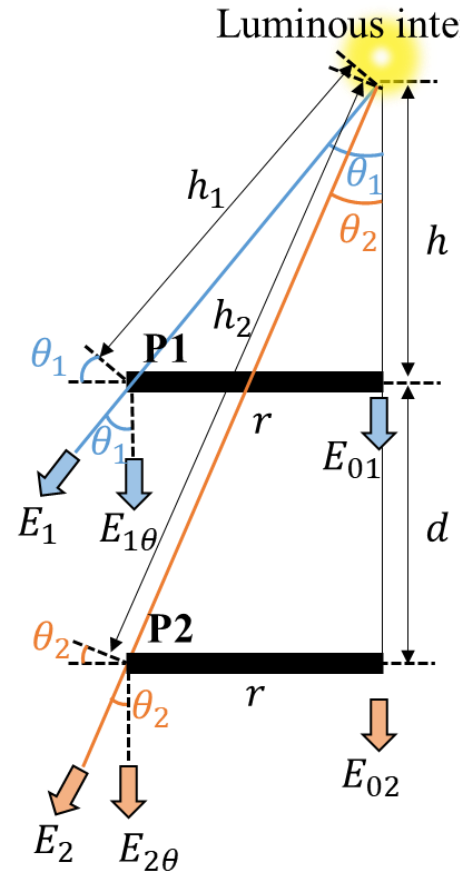

Fig. 2.2 Principle for Detecting Position from Illuminance

Let the distances from the light source to just under the light source be $h$ and $h+d$, respectively. Then, the illuminance of each is expressed as follows.

$$
\begin{aligned}
& E_{01}=\frac{I}{h^{2}} \\
& E_{1}=\frac{I}{h_{1}^{2}} \\
& E_{02}=\frac{I}{(h+d)^{2}} \\
& E_{1}=\frac{I}{h_{2}^{2}}
\end{aligned}
$$

From the cos fourth power law,

$$
\begin{aligned}
& E_{1 \theta}=E_{01} \cos ^{4} \theta_{1} \\
& E_{2 \theta}=E_{02} \cos ^{4} \theta_{2}
\end{aligned}
$$

Substitute Equation (2.10) (2.11) into Equation (2.6) (2.7), respectively.

$$
\begin{gathered}
E_{1 \theta}=\frac{I}{h^{2}} \cos ^{4} \theta_{1} \\
E_{2 \theta}=\frac{I}{(h+d)^{2}} \cos ^{4} \theta_{2}
\end{gathered}
$$

Also, from the Pythagorean theorem

$$
\begin{gathered}
\cos ^{2} \theta_{1}=\frac{1}{1+\frac{r^{2}}{h^{2}}}=\frac{h^{2}}{h^{2}+r^{2}} \\
\cos \theta_{1}=\frac{h}{\sqrt{h^{2}+r^{2}}}
\end{gathered}
$$

Similarly,

$$
\cos \theta_{2}=\frac{h+d}{\sqrt{(h+d)^{2}+r^{2}}}
$$

Then, the calculation in this way.

$$
\cos ^{4} \theta_{1}=\frac{h^{4}}{\left(h^{2}+r^{2}\right)^{2}}
$$




$$
\cos ^{4} \theta_{2}=\frac{(h+d)^{4}}{\left\{(h+d)^{2}+r^{2}\right\}^{2}}
$$

Substitute Equation (2.10) (2.11), and divide.

$$
\frac{E_{1 \theta}}{E_{2 \theta}}=\frac{h^{2}\left\{(h+d)^{2}+r^{2}\right\}^{2}}{\left(h^{2}+r^{2}\right)^{2}(h+d)^{2}}
$$

Solve equation (2.18) as $r^{2}$ equation.

$$
\begin{aligned}
\left\{E_{1 \theta}(h+d)^{2}-\right. & \left.E_{2 \theta} h^{2}\right\} r^{4}+2\left(E_{1 \theta}-E_{2 \theta}\right) h^{2}(h+d)^{2} r^{2} \\
+h^{2}(h+d)^{2}\left\{E_{1 \theta} h^{2}-E_{2 \theta}(h+d)^{2}\right\} & =0
\end{aligned}
$$

therefore

$r^{2}$

$=\frac{-\left(E_{1 \theta}-E_{2 \theta}\right) h^{2}(h+d)^{2} \pm h(h+d) d(d+2 h) \sqrt{E_{1 \theta} E_{2 \theta}}}{E_{1 \theta}(h+d)^{2}-E_{2 \theta} h^{2}}$

Since there is no negative solution, from equation (2.19)

$r^{2}$

$=\frac{-\left(E_{1 \theta}-E_{2 \theta}\right) h^{2}(h+d)^{2}+h(h+d) d(d+2 h) \sqrt{E_{1 \theta} E_{2 \theta}}}{E_{1 \theta}(h+d)^{2}-E_{2 \theta} h^{2}}$

Therefore, the radius with the light source as the origin can be obtained.

\section{Experiment}

It was placed as shown in Fig. 2.3 and the accuracy was measured. The distance from the light source to the first photodiode was $150 \mathrm{~cm}$, and the distance to the second photodiode was $180 \mathrm{~cm}$. The light source was lowered from $70 \mathrm{~cm}$ down by $10 \mathrm{~cm}$ and measured up to $30 \mathrm{~cm}$.

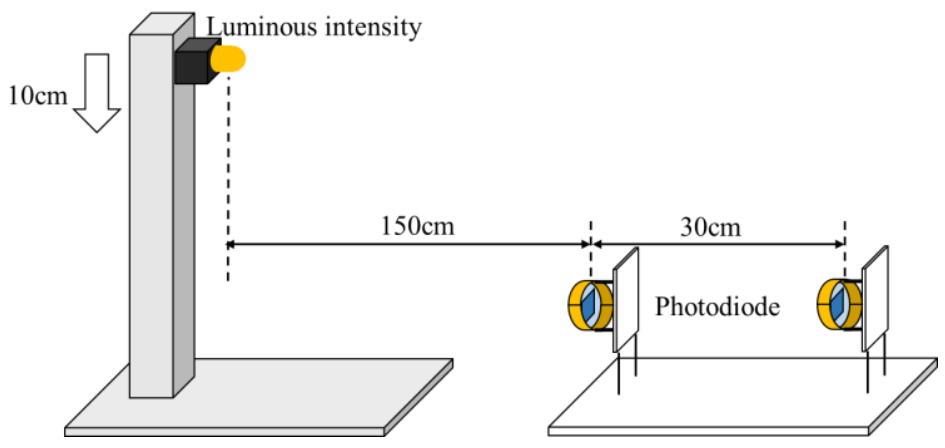

Fig. 3.1 Image of Measurement

The experimental results are shown in Fig. 3.2.

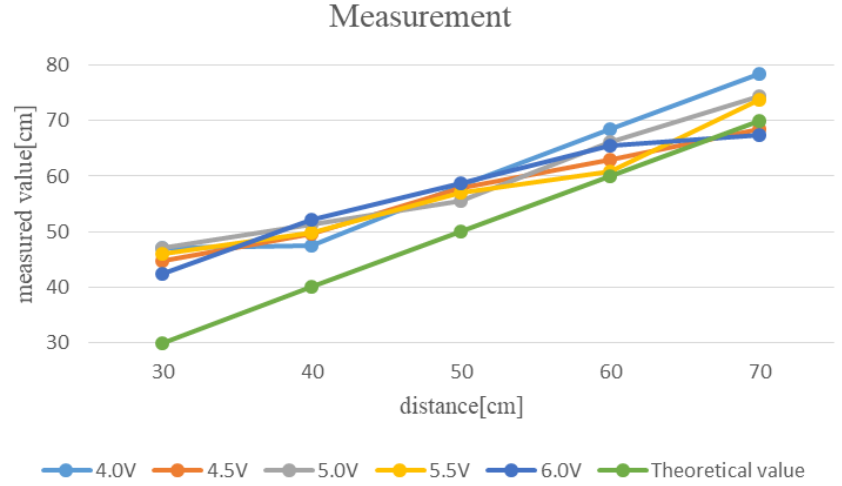

Fig. 3.2 Graph of experiment results

The maximum error at this time is $17.2 \mathrm{~cm}$, the minimum error is $0.9 \mathrm{~cm}$, and the average error is $8.1 \mathrm{~cm}$.

Using the data obtained in section 3.1, we compared it with the conventional method. The experimental results are shown in Fig. 3.4.

\section{Measurement}

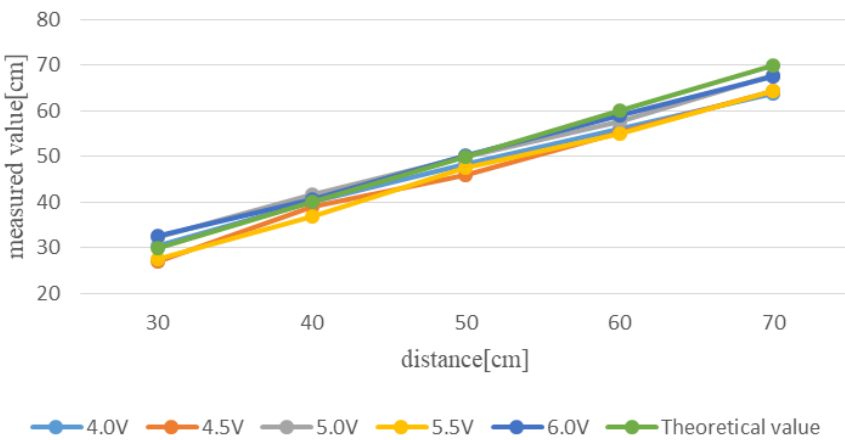

Fig. 3.4 Experimental results of conventional method

The maximum error at this time is $6.3 \mathrm{~cm}$, the minimum error is $0.02 \mathrm{~cm}$, and the average error is 2.61 $\mathrm{cm}$. However, LED illumination has the characteristic that illuminance decreases with time. Therefore, in the conventional method, since the calculation method depends on $E_{\max }$ as the maximum illuminance, the accuracy decreases over time. Figure 3.5 shows the result of measurement when it is assumed that the illuminance has decreased by about $15 \%$ after 1 hour. 


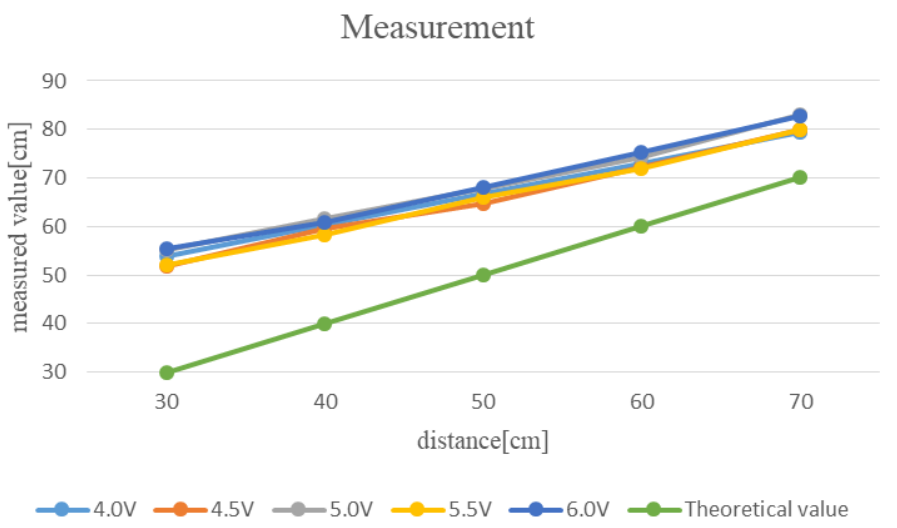

Fig. 3.5 Graph of experimental results under the condition with $85 \%$ illuminance reduced of conventional method

The maximum error at this time is $25.3 \mathrm{~cm}$, the minimum error is $9.4 \mathrm{~cm}$, and the average error is 16.9 $\mathrm{cm}$. Also, a graph obtained by superimposing the proposed method and conventional method is shown in Fig. 3.6.

Measurement

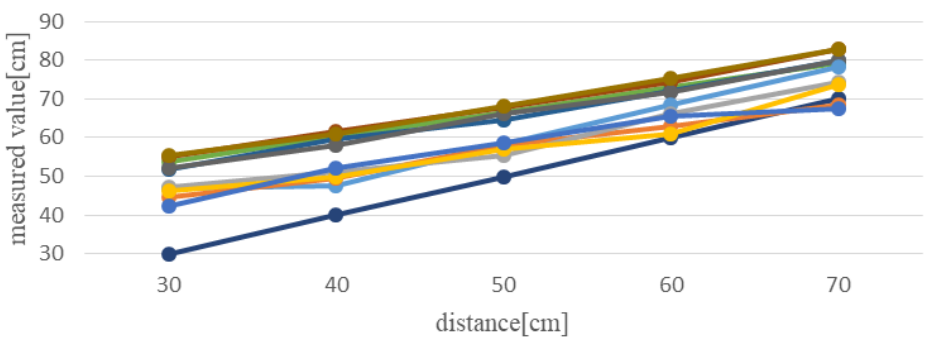

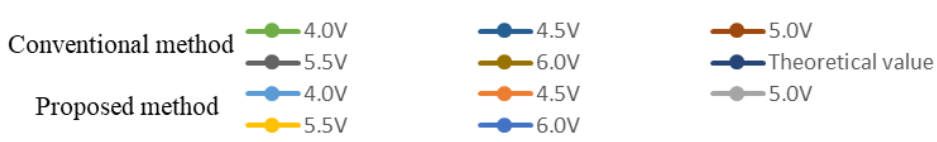

Fig. 3.6 Graph of experimental results of proposed method and conventional method under the condition with $85 \%$ illuminance reduced

Although the accuracy of the conventional method decreases very much as time elapses from Fig. 3.6, it was found that the proposed method was able to perform rough position measurement regardless of the passage of time.

\section{Conclusion and consideration}

From the experimental results, we could develop a system that can measure the position regardless of the passage of time, although the accuracy is not high with respect to the point that the accuracy decreases with the lapse of time which was a problem of the conventional method. The following points can be cited as a consideration regarding accuracy.

- The photodiode nearer to the light source becomes shade and affects the result of the far photodiode.

$\rightarrow$ It is necessary to review the device fixing the photodiode.

- The calculation formula is complicated.

$\rightarrow$ Since an error is included in the obtained illuminance, by using the illuminance many times in the equation, the final error becomes large.

Therefore, in the future, I would like to conduct research by paying attention to the following two points.

- Make an arrangement that does not become shadow.

- Make it possible to measure the position with as simple a formula as possible.

\section{References}

(1) Kanako Kinoshita, Akihiro Ito, Kenta Kagemoto, Mizuho Kimura, Shiyuan Yang: "Location estimation method using photoconductor diode and room lighting", Proceedings of The 6th IIAE International Conference on Industrial Application Engineering 2018 , pp. 270-276, 2018

(2) Shiyuan Yang, Kenta Kagemoto, Kosuke Onishi: "Self-Position Measurement for Indoor Using Ceiling LED Lights", Journal of the Institute of Industrial Applications Engineers Vol.5, No.2, pp.86-89, 2017

(3) Yoshino Masaki, Shinichiro Haruyama, Masao Nakagawa : "High-accuracy positioning system using visible LED lights and image sensor.”, 2008 IEEE Radio and Wireless Symposium. IEEE, pp.439-442, (2008).

(4) Nor Aida Mahiddin, Elissa Nadia Madi, Siti Dhalila, Engku Fadzli Hasan, Suhailan Safie and Noaizan Safie : “User Position Detection In An Indoor Environment.", International Journal of Multimedia and Ubiquitous Engineering, Vol.8, No.5, pp.303-312, (2013) 